\title{
Modèle mathématique de la parturition humaine
}

\section{Le concept d'instabilité critique}

Prédire le moment de la naissance quelques heures ou quelques jours à l'avance avec une bonne précision serait d'une grande utilité, notamment au cours de certaines maladies liées à la grossesse. Cependant, le mécanisme de déclenchement spontané de la parturition implique de nombreux facteurs hormonaux, génétiques et d'environnement, mais aucun des phénomènes décrits à l'heure actuelle ne permet de prévoir de façon fiable l'imminence de l'entrée en travail.

A l'opposé de l'approche traditionnelle qui vise à réduire la complexité à une somme d'éléments simples, nous proposons d'appliquer au phénomène de la parturition les principes de la physique des milieux complexes qui a pour but l'étude de l'émergence de la complexité à l'échelle macroscopique comme résultant de l'interaction répétée entre de nombreux sous-systèmes [13]. Plusieurs autres applications de ces notions et des méthodes mathématiques sous-jacentes ont été développées et ont fait leur preuve dans des domaines aussi divers que l'hydrodynamique, la biologie, la sismologie, etc. Le dénominateur commun de ces systèmes est qu'ils peuvent être représentés par de nombreux sous-systèmes non linéaires couplés entre eux et dont les interactions itérées les conduisent à s'autoorganiser en donnant, dans des conditions favorables, des comportements macroscopiques cohérents. Cette aptitude à la transition progressive d'un état de repos ou d'activité faible vers une activité cohérente définit le concept d'instabilité cri-
L'expérience de Rayleigh-Bénard, ou "problème de la casserole chauffante ", est l'un des systèmes-paradigmes les plus simples de ce type de comportement [4]. Considérons de l'eau placée entre deux plaques maintenues à des températures différentes : une température $T_{0}$ inférieure pour la plaque du haut, une température $\mathrm{T}_{0}+\mathrm{T}$ pour la plaque du bas. Dans tout fluide thermiquement dilatable, une différence de température crée une différence de masse spécifique. Le liquide froid, qui est dense et placé en haut du dispositif, tend à descendre, tandis que le liquide plus chaud et donc moins dense qui se trouve proche du bas a tendance à monter. Tant que la différence de température $T$ reste petite, il n'apparaît aucun mouvement de convection en raison des effets stabilisants de la viscosité et de la conduction thermique du fluide. Il existe une valeur particulière $T_{c}$, dite critique, de la différence de température, pour laquelle une instabilité s'installe: en dessous de $T_{c}$, le fluide est en moyenne au repos et seules existent de petites fluctuations de température, de densité et de vitesse. Au-dessus de $\mathrm{T}_{c}$, un mouvement d'ensemble apparaît, caractérisé par une organisation spatiale remarquable en rouleaux de convection.

\section{Application du modèle d'instabilité critique à la parturition}

Nous proposons que la parturition pourrait être également représentée comme une instabilité critique, donnant lieu à la transition d'une phase de repos, caractérisée par une activité utérine faible et temporellement incohérente (état d'activité de nombreux oscillateurs intermittents et désynchronisés que sont les fibres musculaires lisses), vers un état de contraction globalement cohérent dans le temps et l'espace, selon lequel l'utérus fonctionnerait comme un unique oscillateur macroscopique.

Il peut paraître surprenant qu'un modèle si simple, se focalisant sur l'utérus maternel, puisse décrire l'extrême complexité du système mère-fœetus et leur couplage par le placenta [5-7]. Cette complexité est bien illustrée par le myomètre maternel, qui est un tissu hétérogène formé de plusieurs couches différentes par leur origine embryologique et présentant des propriétés histologiques et contractiles spécifiques [7]. Dans le corps utérin, on doit distinguer les couches externe (longitudinale) et interne (circulaire), composées principalement de cellules musculaires lisses et séparées par une couche intermédiaire riche en tissus conjonctif et vasculaire, mais pauvre en cellules musculaires. Cette complexité qui existe aussi dans l'utérus au niveau moléculaire est caractérisée par l'action des nombreuses substances donnant lieu à des phénomènes d'amplification et d'interaction (cross-talk) qui évolueraient avec le développement et la maturité de l'unité foeto-placentaire.

Dans cette modélisation mathématique de la parturition que nous proposons, le paramètre de contrôle, analogue à la différence de température dans l'expérience de RayleighBénard, est un paramètre de maturité, croissant avec le temps. Le paramètre d'ordre, qui était la vitesse de convection dans l'exemple précité, devient l'amplitude des contractions de l'utérus dans le modèle de la parturition. Ce paramètre d'ordre 
quantifie la notion de transition d'un état de repos, où le paramètre d'ordre est nul en moyenne, vers un état actif où il devient non-nul. Ces idées sont résumées sur la figure 1, qui représente le diagramme de bifurcation, proche du seuil, d'une telle instabilité dite supercritique.

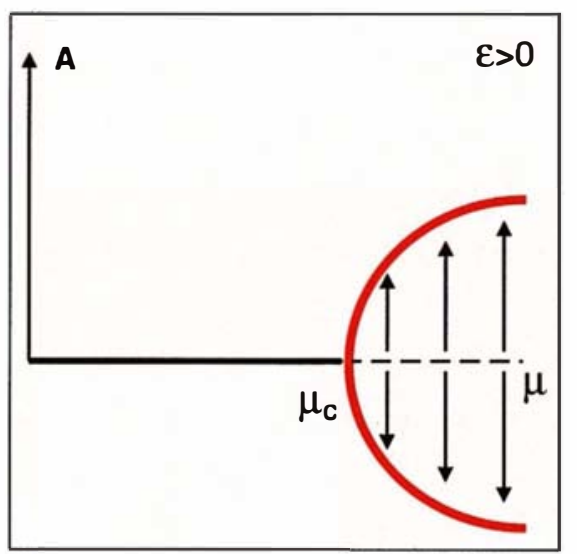

Figure 1. Bifurcation super-critique. $A$ est le paramètre d'ordre de l'activité utérine et $\mu$ le paramètre de contrôle de la maturité. Les lignes continues correspondent au comportement stable, attracteur de la dynamique, tandis que les lignes pointillées représentent les comportements instables. Pour $\mu$ plus petit que $\mu_{c}$ A est attiré vers 0 (activité faible), tandis que $A$ tend vers une valeur finie pour $\mu$ plus grand que $\mu_{c}$. Notons la croissance rapide du paramètre d'ordre A (activité utérine) quand le paramètre de contrôle de la maturité $\mu$ traverse sa valeur critique $\mu_{c}$. Le paramètre de contrôle $\varepsilon$ est un paramètre de sous-corticalité traduisant la possibilité d'une "instabilité retardée" du système mère-fœtus. Lorsque $\varepsilon$ est supérieur à 0 , la parturition n'est pas retardée.

\section{Résumé de la formulation mathéma- tique et ses conséquences}

Le modèle mathématique, dont les aspects plus techniques ont été précédemment décrits [8], est fondé d'abord sur un certain nombre de théorèmes de "réduction" $[9,10]$ qui démontrent que le comportement d'un système complexe, proche d'une bifurcation, peut être décrit par un petit nombre de variables pertinentes régies par des équations différentielles ordinaires dépendant d'un seul paramètre de contrôle, le paramètre de maturation $\mu$ dans le cas qui nous occupe ici. Les équations décrivant l'évolution temporelle de l'activité utérine (A) en fonction de $\mu$ sont représentées par leur solution graphique sur la figure 1 .

L'existence d'une bifurcation critique entraîne d'autres conséquences qui devraient être mesurables. En effet, même en dessous du seuil $\mu_{c}$ de maturation, l'utérus n'est pas complètement au repos. Cela est en accord avec la théorie mathématique de la bifurcation critique qui peut également prédire la manière dont les fluctuations spontanées d'activité augmentent à l'approche de la maturation pour finalement donner lieu au comportement collectif synchronisé que sont les contractions utérines lors de l'accouchement [11]. De plus, le modèle donne la dépendance, en fonction de la maturité, de l'extension spatiale le long de l'utérus des domaines de contractions spontanées, généralement connue sous le nom de longueur de corrélation. Cette longueur de corrélation augmente considérablement à l'approche du terme, traduisant la tendance à la synchronisation de toutes les cellules musculaires lisses de l'utérus.

Le message à retenir de cette formulation est le suivant: à l'approche d'une instabilité ou bifurcation critique, l'activité spontanée incohérente de l'utérus doit augmenter et évoluer d'une manière spécifique. Cela pourrait être l'objet d'observations fines.

De plus, selon ce modèle mathématique, à l'approche de la bifurcation critique, le système mère-fœtus doit devenir de plus en plus "susceptible" et présenter des réponses d'amplitude croissante à un stimulus extérieur. Ainsi, pour une perturbation locale donnée sur l'utérus, le modèle mathématique pourrait prédire la diffusion spatiale dans d'autres régions avec un coefficient de diffusion qui augmente notablement à l'approche de l'instabilité critique, traduisant la grande sensibilité du système mère-fœetus à l'approche du terme.

Ce formalisme mathématique peut également être étendu afin de rendre compte du phénomène de post-maturité dans lequel la grossesse se prolonge au-delà du terme habituel. Dans notre hypothèse, il pourrait correspondre, soit à un simple retard de maturation, le paramètre $\mu$ atteignant le seuil critique $\mu_{c}$ plus tard que d'habitude, soit à une situation pathologique dans laquelle le travail n'est pas déclenché bien que le fotus et le placenta soient arrivés à maturité. La diminution des apports nutritionnels au fœtus via le placenta peut alors aboutir à une souff rance fotale, voire à la mort fotale in utero. On peut formaliser ces aspects pathologiques par l'introduction, outre le paramètre $\mu$ de maturation, d'un second paramètre de contrôle noté $\varepsilon$ traduisant une "instabilité retardée" (dite sous-critique) du système mèrefotus. Avec le paramètre $\varepsilon$, on dispose ainsi d'un moyen de quantifier la tendance du complexe mère-fotus à présenter une post-maturité (figure 2).

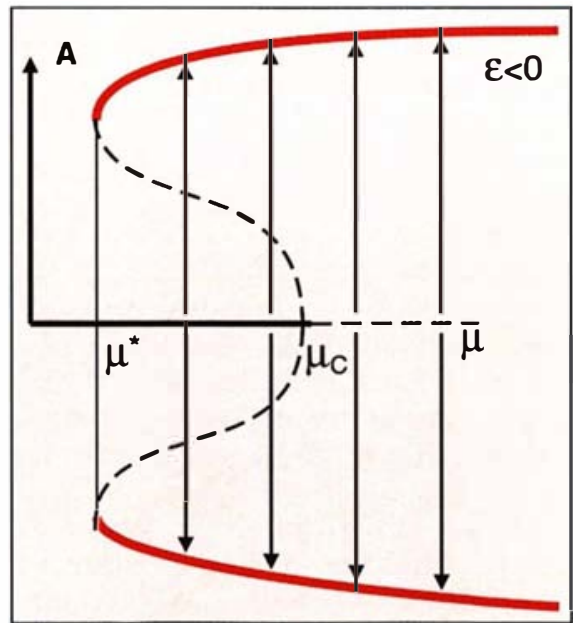

Figure 2. Diagramme de la bifurcation sous-critique [4]. A est à nouveau le paramètre d'ordre de l'activité utérine et $\mu$ est le paramètre de contrôle de la maturité dont $\mu^{*}$ et $\mu_{c}$ représentent deux valeurs critiques. Le paramètre de contrôle $\varepsilon$ négatif indique une parturition retardée. 


\section{Perspectives}

Notre modèle théorique paraît pouvoir rendre compte de divers aspects physiologiques et pathologiques de la parturition humaine. La mise en œuvre d'un protocole expérimental constitue l'étape suivante qui permettrait de mettre en évidence les facteurs pertinents pour définir en particulier le paramètre de maturité $\mu$. Actuellement, les observations obtenues avec les moyens d'étude imparfaits dont nous disposons sont en accord avec ce modèle. En effet la tocographie* externe ou l'électromyographie utérine mettent en évidence des contractions isolées, généralement faibles et intermittentes, précédant le moment de la parturition [12]. Quand le terme approche, on observe une augmentation sensible de l'activité utérine, aussi bien en fréquence qu'en durée et intensité, cette activité culminant pendant le travail, caractérisé par des contractions régulières et synchronisées sur tout l'utérus. D'autres "témoins", notamment biochimiques et moléculaires de la maturation, ont été décrits [13]. C'est finalement l'analyse fine de la contractilité utérine dans son ensemble qui pourrait traduire le plus fidèlement la maturation puisqu'elle intègre tous les sous-systèmes intervenants. Nous avons entrepris un programme de recherche clinique utilisant une méthode d'étude acoustique de la contraction à l'aide de capteurs piézo-électriques. D'autres modèles expérimentaux, tels que la simulation par ordinateur d'un ensemble à deux dimensions de cellules myométriales, pourraient permettre, en modulant la nature du stimulus, son intensité, sa localisation topographique et les paramètres de couplage intercellulaire, de voir s'auto-organiser la propagation de l'activité utérine et d'en déduire les conditions optimales de survenue d'une contractilité synchronisée et régulière telle que celle observée lors de la parturition. Des modèles de ce type ont déjà été développés pour décrire la synchronisation de divers types d'oscillateurs biologiques [3]. Si les prédictions de ce modèle mathématique de bifurcation critique se confirment pour la parturition normale, ses extensions doivent également autoriser l'approche des cas anormaux tels que pré- et postmaturité. On peut noter que le phénomène de transition critique est en fait implicitement considéré dans la pratique obstétricale. Par exemple, l'idée qu'une réduction, même faible, d'un facteur déclenchant tel que l'activité professionnelle puisse suffire à réduire le déclenchement prématuré du travail [14], souscrit à une telle hypothèse de transition critique progressive, caractérisée par une susceptibilité croissante. A l'inverse, dans la grossesse prolongée, une meilleure analyse des paramètres de maturation et de contrôle, respectivement $\mu$ et $\varepsilon$, pourrait permettre de distinguer les cas de grossesses prolongées pathologiques (cas de post-maturité vraie) des grossesses prolongées correspondant à un simple retard de maturation.

La nature fondamentalement coopérative des phénomènes de maturation et de parturition qui est proposée ici peut expliquer les difficultés rencontrées jusqu'à présent pour identifier de manière univoque des paramètres prédictifs dans la succession des événements biochimiques précédant l'entrée en travail. Un phénomène comparable se rencontre dans la structuration progressive des champs de contraintes de la croûte terrestre à l'approche d'un grand tremblement de terre. Pour l'anecdote, c'est le travail de l'un d'entre nous [15] sur ces phénomènes qui a suscité la curiosité d'une de ses collègues impliquée dans la recherche médicale, qui y a vu une analogie (naissance et tremblement de terre?), menant finalement à cette collaboration originale

\section{Didier Sornette}

Laboratoire de physique de la matière condensée, Cnrs URA 190, université de Nice-Sophia Antipolis, BP 71, 06108 Nice Cedex 2, France.

\section{Bruno Carbonne}

Inserm $U .361$, reproduction et physiopathologie obstétricale et maternité PortRoyal - Baudelocque, 123, boulevard de Port-Royal, 75014 Paris, France.

\section{Françoise Ferré}

Inserm $U .361$, reproduction et physiopathologie obstétricale, 123, boulevard de Port-Royal, 75014 Paris, France.

\section{Christian Vauge}

Laboratoire d'instrumentation physique, université Paris XII - Val-de-Marne, bâtiment L2 - IUT de Créteil, 61, avenue du Général-de-Gaulle, 94010 Créteil Cedex, France.

\section{Émile Papiemik}

Maternité Port-Royal - Baudelocque, 123, boulevard de Port-Royal, 75014 Paris, France.

\section{RÉFÉRENCES}

1. Haken $\mathrm{H}$. Advanced synergetics, $2^{\text {nd }}$ ed. Berlin: Springer, 1983

2. Hohenberg PC, Halperin BI. Theory of dynamical critical phenomena. Rev Mod Phys 1977; 49: 435-79

3. Strogatz SH. Norbert Wiener's brain waves. In: Lecture notes in biomathematics, Berlin: Springer, 1993: 100.

4. Bergé P, Pomeau Y, Vidal C. Order within Chaos. New York: Wiley, 1984.

5. Wray S. Uterine contraction and physiological mechanisms of modulation. Am $J$ Physiol 1993; 264 : C1-18. 


\section{RÉFÉRENCES}

6. Doualla-Bell KottoMaka F, Ferré F. Regulation of myometrial contractility in human pregnancy. In: Koppe JC, Eskes TKAB, van Geijn HP, Wiesenhaan PH, Ruys JH, eds. Care, concern and cure in perinatal medicine. New York: The Parthenon Publishing Group, 1993; 19: 131-46.

7. Ferré F, Germain G, Harbon S, Hollingsworth M, Keirse M. Biochemistry and pharmacology of the myometrium. In : Conference Philippe Laudat. Paris: INSERM, 1990: 29-80.

8. Sornette D, Ferré F, Papiernik E. Mathematical model of human gestation and parturition: implications for early diagnosis of prematurity and postmaturity. Int Bifurcation Chaos 1994 ; 4: 693-9.

9. Thom R. Stabilité structurelle et morphogenèse. New York: Benjamin, 1972.

10. Arnol'd V. Chapitres supplémentaires sur la théorie des équations différentielles ordinaires. Moscou: Mir, 1980

11. Sornette D. Sweeping of an instability: an alternative to self-organized criticality to get power laws without parameter tuning. $J$ Phys I France 1994; 4: 209-21.

12. Main DM, Grisso JA, Wold T, Snyder ES Holmes J, Chiu G. Extended longitudinal study of uterine activity among low-risk women. Am J Obstet Gynecol 1992; 1657 . 1317-22.

13. Litime MH, Pointis G, Breuiller M, Cabrol D, Ferré F. Disappearance of $\beta$-adrenergic response of human myometrial adenylate cyclase at the end of pregnancy. $J$ Clin Endocrinol Metab 1989; 69 : 1-6.

14. Papiernik E. Proposals for a programmed prevention policy of preterm births. Clin Obstet Gynecol 1994; 27 : 614-35.

15. Sornette A, Sornette D, Vanneste C. Phénomènes critiques auto-organisés, tectonique des plaques et tremblements de terre. Bull Soc Fr Phys 1991 ; 82: 12-4.

\section{TIRÉS À PART}

D. Sornette 